\title{
Modifier genes in pseudoxanthoma elasticum: novel insights from the Ggcx mouse model
}

\author{
Alain Hovnanian \\ Received: 10 December 2009 / Accepted: 10 December 2009/Published online: 11 February 2010 \\ (C) Springer-Verlag 2010
}

Keywords PXE·Mouse model $\cdot$ Genetics

\section{Clinical and genetic description}

Pseudoxanthoma elasticum (PXE) is an autosomal recessive multisystem disorder affecting the skin, the eyes, and the cardiovascular system characterized by progressive calcification and fragmentation of elastic fibers [1]. It is a rare disease (incidence is estimated in the range of 1 in 50,000-75,000) which shows considerable morbidity and mortality related to ocular and cardiovascular complications. Onset is often in late childhood or adolescence. The skin shows small yellowish papules at flexural areas typically located on the neck, axillae, and antecubital fossae. These lesions progressively coalesce to form larger plaques with cobblestone appearance, skin loses its elasticity, and typical redundant folds develop. Characteristic histological features are fragmentation and calcification of elastic fibers seen on von Kossa or Alizarin red staining. The ocular manifestations consist in angioid streaks resulting from the calcification and cracks of Bruch's membrane, the elastic-rich layer of the retina. As the disease progresses, aberrant vascularization,

A. Hovnanian $(\bowtie)$

Department of Genetics, University Paris V René Descartes,

Necker Hospital for Sick Children, Inserm U563,

149 Rue de Sèvres,

75743 Paris Cedex 15, France

e-mail: alain.hovnanian@inserm.fr

\author{
A. Hovnanian \\ Department of Dermatology, University Paris V René Descartes, \\ Necker Hospital for Sick Children, \\ 149 Rue de Sèvres, \\ 75743 Paris Cedex 15, France
}

proliferation, and retinal hemorrhages (PXE-associated retinopathy) can lead to definitive central visual loss. Visual complications are difficult to treat and have the greatest impact on the functional prognosis of patients with PXE. The cardiovascular manifestations are caused by degeneration of the elastic laminae of medium-sized arteries. They lead to early onset of peripheral vascular occlusive disease, including intermittent claudication, hypertension, and rarely early myocardial infracts, and to gastrointestinal bleeding. PXE shows considerable phenotypic heterogeneity in the onset, course, and expression of the disease, even between patients sharing the same mutation.

\section{PXE is a metabolic disease}

PXE was thought to be a primary disorder of elastic fibers until its causative gene was identified in 2000 [2, 3]. PXE is caused by mutations in the ATP-binding cassette subgroup $\mathrm{C}$ member 6 ( $A B C C 6$ ) gene on chromosomal region 16p13.1. ABCC6 encodes multiresistance-associated protein 6 (MRP6), a member of the large ATP-dependent transmembrane transporter family. The majority of ABCC6 mutations are inactivating mutations leading to the absence of functional MRP6 [4-8]. To date, no correlation has been reported between the nature or position of the mutations and the phenotype, suggesting the role of modifier genes and environmental factors.

A puzzling aspect of PXE is that MRP6 is expressed primarily in the liver, to a lesser extent in the proximal tubes of the kidneys, and at a very low level, if at all, in tissues affected in PXE that is the skin, eyes, and cardiovascular system [9]. This expression pattern has very early raised the question of the pathogenesis of PXE and 
the mechanism by which abnormal mineralization occurs in affected tissues. Two hypotheses have been suggested. A "metabolic" hypothesis postulates that ectopic mineralization results from changes in the levels of circulating factor(s) that are required to prevent excessive mineralization. A "cellular" hypothesis involves a primary connective tissue defect from fibroblasts or smooth muscle cells [10]. In fact, several recent observations strongly support the concept that PXE is a primary metabolic disease with secondary connective tissue manifestations, although the precise biological pathway is still unknown. Serum factors from PXE patients were shown to alter elastin fiber formation in vitro [11]. Moreover, transplantation experiments of muzzle skin from Abbc6 knockout (KO; see below) and wild-type (WT) mice onto WT of KO mice in which mineralization of connective tissue capsule surrounding the bulb of vibrissae was used as an early biological marker [12]. Grafted WT mouse muzzle skin onto KO mice developed early mineralization of the vibrissae, whereas $\mathrm{KO}$ mouse muzzle skin onto WT mice did not. These results very clearly implicate circulating factors as a cause of ectopic mineralization.

\section{Which biological cascade?}

Despite these advances, the precise function of MRP6 and the nature of its substrate are still unknown. MRP6 is localized at the basolateral surface of hepatocytes which suggests, by analogy with other MRPs transporters, that it could serve as an efflux pump transporting metabolites from hepatocytes to the bloodstream. It is thus postulated that MRP6 dysfunction could lead to lack of export of this metabolite in the systemic circulation, which would promote calcification of the target organs. ABCC6 expression in the kidney also suggests that MRP6 may play an important role in regulating the clearance of metabolite(s) involved in mineralization. However, the nature of the substrate and the reasons why only the skin, the eyes, and the cardiovascular system are affected are still not understood. It has been shown that the human MRP6 can transport glutathione conjugates in vitro, but the in vivo MRP6 substrate has remained elusive. The diversity of physiological roles for the $\mathrm{ABC}$ subfamily suggests a unique transport function that is not compensated for by other $\mathrm{ABC}$ transporters.

To gain insight into $A B C C 6$ function in vivo, $\mathrm{KO}$ mice with targeted ablation of the mouse $A b c c 6$ gene have been generated [13, 14]. The $A b c c \sigma^{--}$mice show ectopic calcification of the skin, arterial blood vessels, and retina, which are reminiscent of that in PXE. A striking observation was mineralization of the connective tissue capsule surrounding the bulge of vibrissae as early as 5 weeks of age. Electron microscopy of the skin revealed not only mineralization of elastic fibers but also that of collagen fibers.

\section{GGXC, a link between vitamin K-dependent pathway and mineralization}

Although the nature of this/these factors has not been disclosed yet, several observations point to a possible link with a vitamin $\mathrm{K}$-dependent pathway. This hypothesis came from the report of PXE-like cutaneous changes in a few families with deficiency in vitamin K-dependent coagulation factors [15-18]. Molecular analysis of some of these patients disclosed mutations in the GGCX gene encoding the vitamin $\mathrm{K}$-dependent $\gamma$-glutamyl carboxylase which is essential for post-translational modification of clotting factors and matrix gla protein (MGP). MGP plays a key role in the prevention of the mineralization process and requires $\gamma$-glutamyl carboxylation to be active [19]. Importantly, cMGP is not detected in lesional skin from PXE patients, which supports its possible implication in the abnormal mineralization process seen in the disease (see Fig. 1) $[20,21]$.

\section{Other actors of the mineralization process as potential modifier genes}

While the precise nature of the metabolite(s) transported by MRP6 and the pathway involved are still unknown, another striking feature of PXE is the highly variable clinical picture of PXE with regard to the age of onset and the severity of the skin, eye, and cardiovascular involvement. The highly variable course of the disease and the absence of genotype-phenotype correlation suggest the involvement of modifying genes and the environment. Several studies have tested the possible implication of genes known to influence the mineralization process.

Specifically, levels of serum fetuin-A, a systemic inhibitor of calcification, were found to be lower in PXE patients, although no genetic association of fetuin-A polymorphisms with PXE could be identified [22]. Secreted phosphoprotein 1 (SPP1, previously osteopontin [OPN]), which plays a major role in regulating mineralization processes in various tissues, shows enhanced expression in the dermis of PXE. An association study suggested that polymorphisms in the SPP1 promoter contribute to PXE susceptibility [23]. Genes involved in the response to oxidative stress have also been implicated in PXE: polymorphisms in the xylosyltransferase genes (XT) leading to higher serum XT activity were found to correlate with disease severity in patients with PXE [24]; association studies have suggested that catalase, superoxide dismutase 2 , and glutathione peroxidase 1 are risk factors for early disease onset [25]. More recently, VEGF gene polymorphisms were found to be associated with early and severe retinopathy in PXE [26]. 
Fig. 1 Putative mechanism of ectopic mineralization in PXE and in Ggcx deficiency. Ggcx $\gamma$-glutamyl carboxylase gene, $M G P$ matrix gla protein, vit $\mathrm{KH}_{2}$ reduced vitamin $\mathrm{K}$ (an obligatory cofactor of $\gamma$-glutamyl carboxylase). Readers are referred to Li et al. [1]

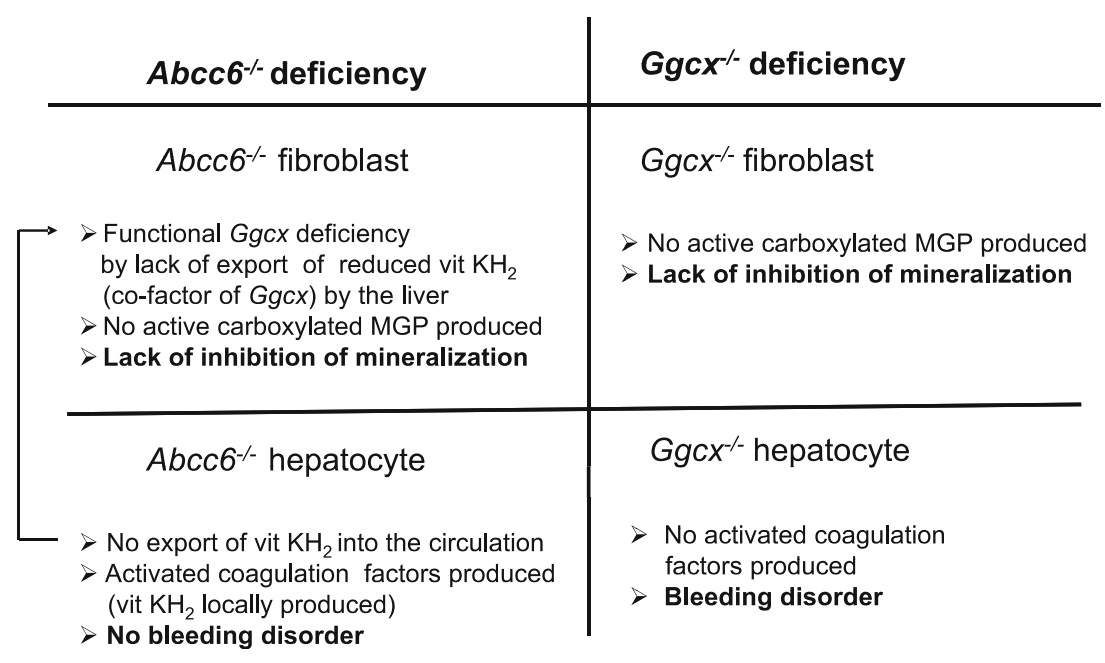

\section{Oxidative stress, GGCX, and vitamin $\mathrm{K}$ cycle}

Oxidative stress and the effect of antioxidant diet have been studied in the $A b c c \sigma^{-1}$ mouse model for PXE [27]. These studies have confirmed the presence of a chronic oxidative stress, but they have shown that antioxidant diet had no effect on ectopic mineralization in the $A b c c \sigma^{1-}$ mouse model. However, oxidative stress leads to the conversion of glutathione (GSH) to its oxidized form GSSG, of which removal from the cell is facilitated by $\mathrm{ABCC} 1$. If such a mechanism is impaired by ABCC6 defects, it is thus possible that the redox potential following oxidative stress is delayed. The vitamin $\mathrm{K}$ cycle is a redox cycle and is sensitive to alterations in the redox potential of the cell [28]. Therefore, alterations in GGCX activity resulting from gene mutations or reduced vitamin $\mathrm{K}$ cycle could have common effects on MGP- $\gamma$-glutamyl carboxylation, leading to undercarboxylation of MGP and ectopic calcification (see Fig. 1). Vitamin $\mathrm{K}$ derivatives, alone or conjugated with glutathione, have thus been suggested as potential effector molecules [27].

Oxidative stress has also been suggested to play a role in acquired PXE-like changes observed in patients with congenital hemolytic anemias such as beta-thalassemia and sickle cell anemia $[29,30]$.These PXE-like alterations are indistinguishable from those of inherited PXE.

\section{The influence of diet on the mineralization process}

Other studies have tested the influence of diet on the mineralization process. The effects of changes in dietary phosphate, calcium, and magnesium on the mineralization process have been studied in $A b c c \sigma^{1-}$ mice using calcification of the connective tissue capsule surrounding the vibrissae as an early phenotypic biomarker [31]. While changes in phosphate did not modify mineralization, enrichment in magnesium (fivefold) significantly reduced connective tissue mineralization in this mouse model of PXE. The use of a phosphate binder or specific mineral modifications (high phosphorus, low calcium, and low magnesium) had no significant effect on ectopic mineralization in the $A b c c \sigma^{1-}$ mice model [32]. These observations suggested that the role of magnesium in particular should be further investigated in PXE patients.

\section{Mineralization in PXE mouse model can be modulated by Ggcx, genetic background, and diet}

In this issue, Jouni Uitto's group has used the $A b c c \sigma^{1-}$ mouse model for PXE and $\mathrm{Ggcx}^{+-}$mice as model systems to explore the influence of genetic and diet determinants on the mineralization process in PXE [33]. They demonstrate very elegantly the role of the Ggcx gene, the genetic background and diet factors in modulating the calcification process in the mouse skin.

To this aim, the authors crossed $A b c c \sigma^{--}$mice with $\mathrm{GgCx}^{+/+}$mice or with $\mathrm{GgCx}^{+/-}$mice and compared the mineralization process. They used ectopic mineralization of the vibrissae as an early biomarker of disease. The degree of mineralization was assessed by computerized morphometric analysis of mineralization of vibrissae after Alizarin red or von Kossa staining and quantified colorimetry of calcium and phosphate deposition. $A b c c \sigma^{1-}$ mice usually develop detectable calcification of whiskers as soon as 5 weeks. Surprisingly, they observed that connective tissue mineralization in $\mathrm{Abcc \sigma}^{1-} / \mathrm{Ggcx}^{+/+}$mice is delayed in comparison with $A b c c \sigma^{-1-}$ mice. In addition, when $A b c c \sigma^{/-}$mice were crossed with $\mathrm{GgCX}^{+-}$mice, their offspring developed ectopic mineralization as early as the $A b c \sigma^{/-}$mice. These results suggested that the $\mathrm{GgCx}^{+/+}$ genetic background is protective from the mineralization process and that Ggcx deficiency in $A b c c \sigma^{-1}$ mice 
accelerates the mineralization process, despite the protective genetic background of the $\mathrm{Ggcx}^{+/-}$strain.

To gain insights into these observations, the authors compared the genetic backgrounds of each mouse strain. The $A b c c \sigma^{-1}$ and $\mathrm{Ggcx}^{+/+}$mice strains resulted from five generation backcrosses with C57BL6/J mice, but the embryonic stem cells which were used to generate the $G g c x$ strain differed from the ones used to generate the $A b c c \sigma^{/-}$ mice by a $129 \mathrm{X} 1 / \mathrm{Svj}$ origin. Therefore, the two mouse strains differed by the contribution of a $129 \times 1 / \mathrm{Svj}$ background in the $\mathrm{Ggcx}^{+/+}$mice. To confirm the influence of this genetic background, the authors crossed the $A b c c \sigma^{1-}$ mice with WT mice with $129 \mathrm{X} 1 / \mathrm{Svj}$ background. They showed that mineralization is delayed in $A b c c \sigma^{--}$mice with a contribution from the $129 \mathrm{X} 1 / \mathrm{Svj}$, thus providing evidence that this $129 \mathrm{X} 1 / \mathrm{Svj}$ background indeed protects from the mineralization process.

Next, the authors studied the influence of diet on the mineralization process. They previously showed that high dietary magnesium prevented calcification [31]. Here, they show that low magnesium diet aggravates calcium deposition in the model for PXE. These findings support the notion that changes in the magnesium level can affect the onset and extent of abnormal mineralization in PXE. They suggest that the role of dietary magnesium in PXE patients is further investigated.

The authors conclude that the 129X1/Svj background delays premature mineralization and that haploinsufficiency for the Ggcx gene accelerates the mineralization process. They also provide evidence that diet can modulate abnormal mineralization.

\section{Why are these results important?}

These results are important because they demonstrate that mineralization in a PXE mouse model can be modulated by genetic factors and diet.

Specifically, they disclose the effect of mouse genetic background on the mineralization process. By identifying the $129 \mathrm{X} 1 / \mathrm{Svj}$ strain as a protective background, they offer the possibility to use backcrossing of $A b c c \sigma^{--}$mice with the $129 \mathrm{X} 1 / \mathrm{Svj}$ strain to generate congenic strains in order to map candidate chromosomal regions and to identify candidate genes protective for PXE. This has high potential to identify the biological pathway involved in the disease and also point to therapeutic targets to reduce or prevent the mineralization process.

The results obtained with the $A b c c \sigma^{/-}$mice with Ggcx haploinsufficiency confirm the implication of the Ggcx gene in the mineralization process. They also validate these mice as animal models suitable for the search of modifying genes in PXE. Together with the recent observations of patients with PXE-like cutaneous mineralization resulting from global vitamin K-dependent coagulation factor deficiency due to GGCX mutations, they further support a possible role of defective export of vitamin $\mathrm{K}$ or vitamin $\mathrm{K}$ derivatives from the liver into the bloodstream.

These results highlight a key role played by vitamin $\mathrm{K}$ in regulating the mineralization process through the activation of calcification inhibitors. As mentioned above, such inhibitors include MGP whose activation by vitamin Kdependent $\gamma$-glutamate carboxylation is essential for the regulation of the mineralization process. Although patients with global coagulation factor deficiency due to GGCX mutations develop PXE-like skin changes, they do not seem to develop eye or cardiovascular lesions which are typical for PXE, suggesting that these tissues may somehow compensate for the abnormal mineralization process in the absence of vitamin $\mathrm{K}$ derivatives. Possible compensation may involve expression of other $\mathrm{ABC}$ transporter proteins, although the nature of $\mathrm{ABCC}$ substrates is usually specific of the type of transporters.

Finally, the study by Li and Uitto [33] is an important step towards a better understanding of the genetic and environmental factors which control the mineralization process in PXE. This article identifies a modifying gene $(G g c x)$, discloses the protective effect of a specific genetic background in mice, and demonstrates for the first time that mineralization in a PXE mouse model can be modulated by genetic factors and diet. By generating new mouse models for genetic dissection of PXE disease mechanism, these studies certainly contribute to the identification of the missing link between ABCC6 deficiency and abnormal mineralization observed in PXE patients. Deciphering the biological cascade clearly will have implications for the development of therapeutic interventions targeting specific abnormalities. These animal models will allow testing the efficiency of vitamin K-based treatment to prevent or reduce tissue mineralization in PXE. Uncovering the pathway(s) involved in the pathogenesis of PXE holds the promise to provide therapeutic targets/targets for therapeutic intervention for this intractable disorder.

\section{References}

1. Li Q et al (2009) Pseudoxanthoma elasticum: clinical phenotypes, molecular genetics and putative pathomechanisms. Exp Dermatol 18:1-11

2. Le Saux O et al (2000) Mutations in a gene encoding an $A B C$ transporter cause pseudoxanthoma elasticum. Nat Genet 25:223227

3. Ringpfeil F, Lebwohl MG, Christiano AM, Uitto J (2000) Pseudoxanthoma elasticum: mutations in the MRP6 gene encoding a transmembrane ATP-binding cassette (ABC) transporter. Proc Natl Acad Sci USA 97:6001-6006 
4. Le Saux O et al (2001) A spectrum of ABCC6 mutations is responsible for pseudoxanthoma elasticum. Am J Hum Genet 69:749-764

5. Pfendner EG et al (2007) Mutation detection in the ABCC6 gene and genotype-phenotype analysis in a large international case series affected by pseudoxanthoma elasticum. J Med Genet 44:621-628

6. Chassaing $\mathrm{N}$ et al (2005) Pseudoxanthoma elasticum: a clinical, pathophysiological and genetic update including 11 novel ABCC6 mutations. J Med Genet 42:881-892

7. Ringpfeil F, Pulkkinen L, Uitto J (2001) Molecular genetics of pseudoxanthoma elasticum. Exp Dermatol 10:221-228

8. Schulz V et al (2006) Mutational analysis of the ABCC6 gene and the proximal $\mathrm{ABCC} 6$ gene promoter in German patients with pseudoxanthoma elasticum (PXE). Hum Mutat 27:831

9. Matsuzaki Y et al (2005) Tissue-specific expression of the ABCC6 gene. J Invest Dermatol 125:900-905

10. Jiang Q, Uitto J (2006) Pseudoxanthoma elasticum: a metabolic disease? J Invest Dermatol 126:1440-1441

11. Le Saux O et al (2006) Serum factors from pseudoxanthoma elasticum patients alter elastic fiber formation in vitro. J Invest Dermatol 126:1497-1505

12. Jiang $Q$ et al (2009) Pseudoxanthoma elasticum is a metabolic disease. J Invest Dermatol 129:348-354

13. Klement JF et al (2005) Targeted ablation of the abcc6 gene results in ectopic mineralization of connective tissues. Mol Cell Biol 25:8299-8310

14. Gorgels TG et al (2005) Disruption of Abcc6 in the mouse: novel insight in the pathogenesis of pseudoxanthoma elasticum. Hum Mol Genet 14:1763-1773

15. Li Q et al (2009) Co-existent pseudoxanthoma elasticum and vitamin K-dependent coagulation factor deficiency: compound heterozygosity for mutations in the GGCX gene. Am J Pathol 174:534-540

16. Rongioletti F, Bertamino R, Rebora A (1989) Generalized pseudoxanthoma elasticum with deficiency of vitamin Kdependent clotting factors. J Am Acad Dermatol 21:1150-1152

17. Le Corvaisier-Pieto $C$ et al (1996) Generalized pseudoxanthoma elasticum combined with vitamin $\mathrm{K}$ dependent clotting factors deficiency. Ann Dermatol Venereol 123:555-558

18. Vanakker OM et al (2007) Pseudoxanthoma elasticum-like phenotype with cutis laxa and multiple coagulation factor deficiency represents a separate genetic entity. J Invest Dermatol 127:581-587

19. Shearer MJ (2000) Role of vitamin K and Gla proteins in the pathophysiology of osteoporosis and vascular calcification. Curr Opin Clin Nutr Metab Care 3:433-438
20. Li Q, Jiang Q, Schurgers LJ, Uitto J (2007) Pseudoxanthoma elasticum: reduced gamma-glutamyl carboxylation of matrix gla protein in a mouse model $\left(A b c c \sigma^{-1}\right)$. Biochem Biophys Res Commun 364:208-213

21. Gheduzzi D et al (2007) Matrix Gla protein is involved in elastic fiber calcification in the dermis of pseudoxanthoma elasticum patients. Lab Invest 87:998-1008

22. Hendig D et al (2006) Role of serum fetuin-A, a major inhibitor of systemic calcification, in pseudoxanthoma elasticum. Clin Chem $52: 227-234$

23. Hendig D et al (2007) SPP1 promoter polymorphisms: identification of the first modifier gene for pseudoxanthoma elasticum. Clin Chem 53:829-836

24. Schon $\mathrm{S}$ et al (2006) Polymorphisms in the xylosyltransferase genes cause higher serum XT-I activity in patients with pseudoxanthoma elasticum (PXE) and are involved in a severe disease course. J Med Genet 43:745-749

25. Zarbock R et al (2007) Pseudoxanthoma elasticum: genetic variations in antioxidant genes are risk factors for early disease onset. Clin Chem 53:1734-1740

26. Zarbock R et al (2009) Vascular endothelial growth factor gene polymorphisms as prognostic markers for ocular manifestations in pseudoxanthoma elasticum. Hum Mol Genet 18:3344-3351

27. Li Q, Jiang Q, Uitto J (2008) Pseudoxanthoma elasticum: oxidative stress and antioxidant diet in a mouse model $\left(A b c \sigma^{-1}\right)$. $\mathrm{J}$ Invest Dermatol 128:1160-1164

28. Shearer MJ, Newman P (2008) Metabolism and cell biology of vitamin K. Thromb Haemost 100:530-547

29. Hamlin $\mathrm{N}$ et al (2003) Acquired pseudoxanthoma elasticum-like syndrome in beta-thalassaemia patients. Br J Haematol 122:852854

30. Fabbri E, Forni GL, Guerrini G, Borgna-Pignatti C (2009) Pseudoxanthoma elasticum-like syndrome and thalassemia: an update. Dermatol Online J 15:7

31. LaRusso J, Li Q, Jiang Q, Uitto J (2009) Elevated dietary magnesium prevents connective tissue mineralization in a mouse model of pseudoxanthoma elasticum $\left(A b c c 6\left({ }^{-/}\right)\right)$. J Invest Dermatol 129:1388-1394

32. LaRusso J, Jiang Q, Li Q, Uitto J (2008) Ectopic mineralization of connective tissue in $\mathrm{Abcc}^{/-}$mice: effects of dietary modifications and a phosphate binder - a preliminary study. Exp Dermatol 17:203-207

33. Li Q, Uitto J (2010) The mineralization phenotype in $A b c c \sigma^{-/}$mice is affected by Ggcx gene deficiency and genetic background-a model for pseudoxanthoma elasticum. J Mol Med (this issue/ s00109-009-0522-8) 